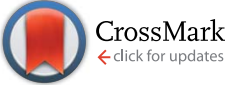

Cite this: RSC Adv., 2017, 7, 543

\title{
Environmental stimuli-responsive self-repairing waterbased superhydrophobic coatings
}

\begin{abstract}
Kunlin Chen, Kun Gu, Siyu Qiang and Chaoxia Wang*
Superhydrophobic coatings have received great attention over the past decades because of their specific performances, but most of them may lose their superhydrophobicity under harsh conditions especially in outdoor applications. In this paper, UV and $\mathrm{pH}$ dual stimuli-responsive self-repairing waterbased superhydrophobic coatings were prepared based on $\mathrm{TiO}_{2}$ with photocatalysis and $\mathrm{pH}$-responsive microcapsules, which were durable against UV irradiation and chemically stable in contact with alkaline or acidic aqueous solutions. These coatings are an environment-friendly waterborne system and could be easily coated on various substrates. What is more, they can restore their superhydrophobicity and self-cleaning ability under UV light even after mechanically damaged or contaminated with organics. Moreover, this superhydrophobic coating was immersed into $5 \mathrm{wt} \% \mathrm{NaCl}$ aqueous solutions for $24 \mathrm{~h}$ or underwent ten icing/melting cycles, their surfaces could also recover the superhydrophobicity under $\mathrm{pH}$ or UV stimuli.
\end{abstract}

Received 12th October 2016 Accepted 11th November 2016

DOI: 10.1039/c6ra25135h

www.rsc.org/advances

\section{Introduction}

Superhydrophobic surfaces are generally defined as surfaces which have a static water contact angle (WCA) larger than $150^{\circ}$ and a slide angle (SA) less than $10^{\circ} .^{1-5}$ The design and preparation of superhydrophobic surface materials with long-term durability is a challenging yet highly rewarding endeavour that contributes to the development of superhydrophobic surfaces for practical applications. ${ }^{6-11}$ Though many efforts have been made to obtain mechanically durable and robust superhydrophobic surfaces, from a practical point of view, introducing a self-repairing ability into artificial super-hydrophobic coatings that are used for protecting against strong sunlight, mechanical wear or organic contamination would be the best strategy to realize the high durability of superhydrophobic surfaces. ${ }^{12-19}$ Therefore, developing a facile method for fabricating self-repairing superhydrophobic surfaces becomes an urgent demand.

Several works about self-repairing superhydrophobic surfaces have been reported recently. For instance, Li et al. fabricated a self-healing superhydrophobic coating by spraying layer-by-layer (LbL) assembly of poly(allylamine hydrochloride)sulfonated poly(ether ether ketone), poly(acrylic acid) and healing agents, such as perfluorooctanesulfonic acid lithium salt (PFOS) or fluoroalkyl silane (POTS), on the surfaces of substrates..$^{20}$ When the surface of this polyelectrolyte coating was damaged, the stored POTS or PFOS in the coating could migrate to the damaged surface for healing the wettability

Key Laboratory of Eco-Textiles, Ministry of Education, School of Textiles and Clothing, Jiangnan University, Wuxi 214122, China. E-mail:wangchaoxia@sohu.com under a minor humid condition. Esteves et al. reported a selfreplenishing superhydrophobic coating consisting of a dispersion consisting of dual-sized silica nanoparticles and fluoroalkyl-terminated polymeric dangling chains. ${ }^{21}$ The surface chemical compositions of the damaged coating can be spontaneously restored by re-orientating the dangling chains towards the surfaces and its superhydrophobicity recovered. Manna et al. prepared easily repairable superhydrophobic porous polymer films through the LbL assembly of branched poly(ethyleneimine) and the amine-reactive polymer poly(vinyl4,4-dimethylazlactone). ${ }^{22}$ When the film was severely crushed, the film could recover superhydrophobicity by water-assisted approach owing to the self-regeneration of topographic structures of porous polymer films. Besides, some researchers successfully fabricated the robust and self-healing superhydrophobic coatings by using silicone rubber and polydimethylsiloxane (PDMS). ${ }^{23-26}$ Owing to the automatic migration of PDMS, the damaged surfaces could be repaired and the hydrophobicity or superhydrophobicity of the coatings recovered. Lately, Lv et al. also reported a new self-healing superhydrophobic surface by creating lotus-leaves-like microstructure on the epoxy shape memory polymer (SMP) ${ }^{27}$ Because the SMP has the special shape memory ability, the crushed microstructure and damaged surface chemistry of the destroyed surfaces could be simultaneously repaired, and the lowadhesive super-hydrophobicity was recovered after a simple heating process. However, to date, in artificial superhydrophobic surfaces applications, the dispersion of hydrophobic substance generally contains pungent or volatile solvents, such as ethanol, toluene and acetone. In spite of their functional advantages, organic solvents are environmentally 
undesirable in large scale industrial processes. ${ }^{\mathbf{2 8 , 2 9}}$ Water-based dispersions are environmentally benign and pose little environmental concern. ${ }^{30}$ Therefore, fabrication of waterborne longlived superhydrophobic surfaces is a recent research endeavour.

In order to achieve this objective, two crucial points must be met: (i) the acquisition of water-based dispersion of low-surface energy materials; (ii) the method to initiate healing and ensure the long time for healing under the outside environment. In view of these requirements, stimuli responsive microcapsules, which can release the encapsulated substances to provide the desired outcome upon external stimuli including $\mathrm{pH}$, temperature, light and magnetic field treatment, ${ }^{31-36}$ may be the best choice to address aforementioned crucial points. Recently, we have successfully prepared the all-water-based self-healing superhydrophobic coatings based on UV-responsive microcapsules. ${ }^{37}$ This coating was a waterborne system, environmentallyfriendly and especially attractive for outdoor applications. Mostly, the damaged surface could recover its superhydrophobic and self-cleaning ability under UV light because of hydrophobic fluoroalkyl silane molecules releasing from UVresponsive microcapsules.

Herein, we developed environmentally-stimuli responsive self-repairing waterborne superhydrophobic coatings by using $\mathrm{pH}$-responsive microcapsules and $\mathrm{TiO}_{2}$ nanoparticles. Scheme 1 shows the schematic diagram of the fabrication method. We firstly prepared fluoroalkyl silane (FAS13)-loaded pH-responsive microcapsules ( $\mathrm{pH}$-capsules) via miniemulsion polymerization. The obtained FAS13-loaded pH-capsules were used as the reservoir of hydrophobe, which can release FAS13 under the acid environment. Then prepared $\mathrm{pH}$-capsules mixed with $\mathrm{TiO}_{2}$ nanoparticles, hydrophobic materials modified nanosilica and waterborne resin to get the self-healing waterborne superhydrophobic coatings. These coatings can be easily coated on various substrates and formed superhydrophobic surface after UV irradiation. More significantly, they can recover their superhydrophobicity and self-cleaning ability under UV light even after mechanically damaged or contaminated with oil. Furthermore, this superhydrophobic coating also has anticorrosion and anti-icing abilities in the extreme natural environments.

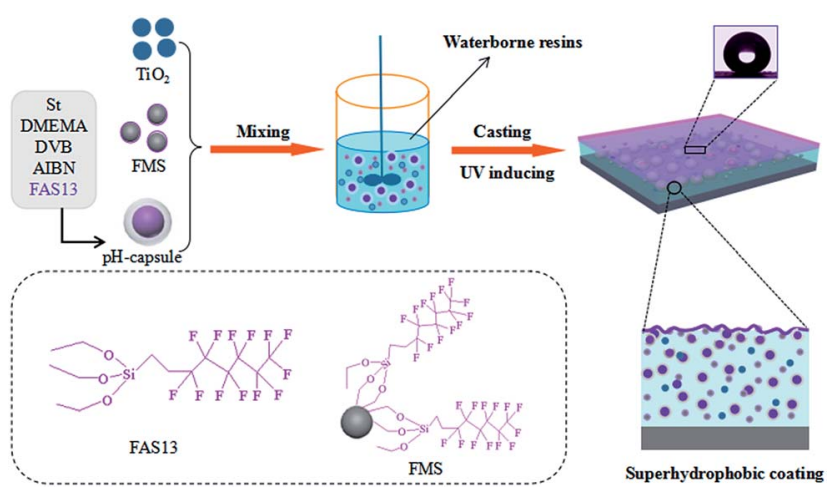

Scheme 1 Schematic illustration of the fabrication of self-repairing waterborne superhydrophobic coatings.

\section{Materials and method}

\subsection{Materials}

Styrene (St, $\geq 99 \%$ ), divinylbenzene (DVB, $\geq 80 \%$ ), ammonia solution (25\%), 2-(N,N-diethylamino)ethyl methacrylate (DEAEMA, $>99 \%)$, acetic acid $(\geq 99.7 \%)$ and ethanol $(\geq 99.7 \%)$ were purchased from Sinopharm Chemical Reagent Co., Ltd. (China). Sodium dodecyl-benzenesulfonate (SDBS, 90\%) and azodiisobutyronitrile (AIBN, 98\%) were bought from Aladdin Chemical Reagent Co., Ltd. (China). Perfluorooctyltriethoxysilane (FAS13) was purchased from Xeogia Fluorine-Silicon Chemical Co., Ltd. (China). St, DEAEMA and DVB were distilled under vacuum before use and the other reagents were used as received. Polysiloxane latex (BS-45, solid content: $50 \mathrm{wt} \%$ ) was purchased from Wacker Chemicals (Germany). Titania nanoparticles (P25, primary size: $25 \mathrm{~nm}$ ) were purchased from Degussa (Germany). Silica nanoparticles (hydrophilic, primary size: $20 \mathrm{~nm}$ ) were purchased from Shanghai Cabot Chemical Co., Ltd. (USA). Deionized water was used throughout the whole experiments.

\subsection{Syntheses of pH-responsive microcapsules}

$1 \mathrm{~g} \mathrm{St}, 1 \mathrm{~g}$ DEAEMA, $0.2 \mathrm{~g}$ DVB, $1 \mathrm{~g}$ FAS13 and $0.06 \mathrm{~g}$ AIBN were added into a $150 \mathrm{~mL}$ round-bottom flask to form a homogeneous solution. Then $100 \mathrm{~mL}$ deionized water and $0.1 \mathrm{~g}$ SDBS were added to the mixture and ultrasonically emulsified for $20 \mathrm{~min}$. The resulting emulsion was purified with a $\mathrm{N}_{2}$ flow for $30 \mathrm{~min}$ and kept stirring at $75{ }^{\circ} \mathrm{C}$ for $24 \mathrm{~h}$ to obtain the microcapsules. The microcapsules solution were further separated by centrifugation at $10000 \mathrm{rpm}$ for $10 \mathrm{~min}$ and then washed with water and ethanol two times. Subsequently, the capsules were re-dispersed in an aqueous solution for further use. One control capsule without DEAEMA was fabricated using the method described above.

\subsection{Preparation of hydrophobic $\mathrm{SiO}_{2}$ nanoparticles}

Briefly, $2 \mathrm{~g}$ hydrophilic silica nanoparticles, $0.5 \mathrm{~g}$ ammonia, and $150 \mathrm{~g}$ water were added to a $250 \mathrm{~mL}$ round-bottom flask and stirred for $30 \mathrm{~min}$. Subsequently, the homogeneous solution containing $10 \mathrm{~g}$ ethanol and $0.3 \mathrm{~g}$ FAS13 were added to the silica nanoparticles solution, and then the mixed solution was stirred at $65{ }^{\circ} \mathrm{C}$ for $20 \mathrm{~h}$ to obtain FAS13-modified silica nanoparticles (FMS). The FMS were then collected by centrifugation (8000 rpm, $10 \mathrm{~min}$ ), washed with water and ethanol, and redispersed in deionized water for further use.

\subsection{Preparation of waterborne superhydrophobic coatings}

In a typical fabrication process, $16 \mathrm{~g}$ pH-capsule aqueous dispersion (solid content: $15 \mathrm{wt} \%$ ), $2.8 \mathrm{~g}$ FMS aqueous dispersion (solid content: $65 \mathrm{wt} \%$ ), $0.3 \mathrm{~g}$ P25 and $10 \mathrm{~g}$ silicone latex (solid content: $50 \mathrm{wt} \%$ ) were mixed at $800 \mathrm{rpm}$ for $10 \mathrm{~min}$ and then deposited on substrates using a drawdown $\operatorname{rod}(120 \mu \mathrm{m})$ and dried at $80{ }^{\circ} \mathrm{C}$ for $15 \mathrm{~min}$. For comparison, four control coatings were also prepared with BS45/P25, BS45/P25/FMS, $\mathrm{BS} 45 / \mathrm{P} 25 / \mathrm{pH}$-capsules, or BS45/FMS/pH-capsules using the same process. 


\subsection{Characterization}

The morphologies of the microcapsules were characterized by a scanning electron microscope (SEM, Sigma HD field, Zeiss, Germany) at an accelerating voltage of $5 \mathrm{kV}$ and a transmission electron microscope (TEM, JEM-2100, JEOL Ltd., Japan) at an accelerating voltage of $20 \mathrm{kV}$. The particle size distribution of microcapsules was measured by dynamic light scattering (DLS) method using Nano-ZS 90 (Malvern, UK). Fourier transform infrared spectroscopy (FTIR) spectra was recorded using an infrared spectrometer (Nicolet Nexus 470, ThermoFisher Corp. USA), with a resolution of $0.5 \mathrm{~cm}^{-1}$ and an accumulation of 32 scans. The dried samples were blended with $\mathrm{KBr}$ to form sample plates. The water contact angle (WCA) and sliding angle (SA) were measured by optical contact angle measuring instrument (Dataphysics, Germany) with a $5 \mu \mathrm{L}$ deionized water droplet at ambient temperature. The average value from five parallel measurements was adopted. The sample coatings were tested in a QUV accelerated weathering tester (QUV/se, Q-Panel Co., Ltd., USA), using UV lamps with a wavelength of $310 \mathrm{~nm}$. The accelerated weathering cycle was set as follows: UV-irradiation at $60{ }^{\circ} \mathrm{C}$ for $4 \mathrm{~h}$ and irradiation intensity of $0.71 \mathrm{~W} \mathrm{~m}^{-2}$, and condensation at $50{ }^{\circ} \mathrm{C}$ for $4 \mathrm{~h}$. The surface composition of coating film was measured by X-ray photoelectron spectroscopy (XPS, Perkin-Elmer PHI 5000C ECSA) using the Al K $\alpha$ line as the excitation source at a $90^{\circ}$ take-off angle. All the binding energy values were calibrated using the reference peak of C1s at $284.6 \mathrm{eV}$.

The abrasion resistance of the superhydrophobic surfaces was evaluated by a homemade polish tester, which contains a piece of 320-grit sandpaper and a weight of $500 \mathrm{~g}$. The sample had a contact area of $2.25 \times 2.25 \mathrm{~cm}^{2}$ with the underlying sandpaper, and was dragged in one direction with a speed of $1 \mathrm{~cm} \mathrm{~s}^{-1}$.

The resistance of coating surfaces to oily contaminants was evaluated by using salad oil as the model pollutants. A thin layer of salad oil was sprayed on the coating surface, and then the coating was put into the QUV accelerated weathering tester. The WCAs were measured after $12 \mathrm{~h}$ interval.

The anti-icing test was carried out in a refrigerator $\left(-10^{\circ} \mathrm{C}\right)$. The water droplets $(\sim 60 \mu \mathrm{L})$ dripped on the sample, and then it was placed in the refrigerator for cooling. This test was repeated for ten times in order to assess the durability against periodic icing/melting. Finally, the sample was put into the QUV accelerated weathering tester for $36 \mathrm{~h}$. WCA and SA were measured during this process.

The ice adhesion strength of the coatings was measured by a pull off adhesion tester (PosiTest AT, DeFelsko Corp., USA). Aluminum dollies with a surface diameter of $20 \mathrm{~mm}$ were etched with a dilute $\mathrm{HCl}$ solution to obtain a hydrophilic surface. One drop of deionized water was dropped on the coated panel, and then a dolly was covered on the water drop, forming a thin layer of water (about $150 \mu \mathrm{m}$ in thickness) between the aluminum dolly and the samples. The panel, together with the dolly was kept in a temperature-controlled chamber (GDWJS250, Shanghai Maijie Test Equipment Ltd., China) at $-10{ }^{\circ} \mathrm{C}$ for at least $15 \mathrm{~min}$. A pull test was then quickly carried out with the adhesion tester in the chamber. The adhesion strength was measured for at least five parallel pulls.

\section{Results and discussion}

\subsection{Preparation of $\mathrm{pH}$-responsive microcapsules}

FAS-Loaded pH-responsive microcapsules were prepared via miniemulsion polymerization of St, DEAMA, DVB and FAS13. Fig. $1 \mathrm{a}$ and $\mathrm{b}$ shows the SEM and TEM images of the as-obtained microcapsules. From these pictures we could see that the microcapsules were regular spheres which had a tint nucleus and a fuscous shell, and the size of microcapsules was about 250-300 nm. DLS analysis also displayed that the mean size of microcapsules was $290 \mathrm{~nm}$, consistent with SEM and TEM images. And the average diameter of microcapsules increased to $420 \mathrm{~nm}$ after acidization at $\mathrm{pH}=3$ for $10 \mathrm{~min}$ (Fig. 1c), suggesting that these microcapsules have the $\mathrm{pH}$ stimulative responsibility. This is mainly because the amino groups of polymer shells of microcapsules could be protonated at $\mathrm{pH}=3$, and then the electrostatic interactions of the positively charged polymer chains made the microcapsules swell. ${ }^{35}$ These protonated microcapsules were further dried and washed using ethanol for three times, and the extracted ethanol was characterized by the FT-IR. As shown in Fig. 1d, a peak at $1205 \mathrm{~cm}^{-1}$ assigned to $\mathrm{C}-\mathrm{F}$ stretching vibration was revealed in the spectrum of pH-responsive microcapsules, while no peak was observed at this wavelength in the unresponsive microcapsules. The FT-IR results proved the $\mathrm{pH}$-induced release of FAS13 from the swollen microcapsules.

\subsection{Preparation of waterborne superhydrophobic coatings}

The waterborne polysiloxane latex formed the dried coating surface was hydrophilicity $\left(\mathrm{WCA}=82.6^{\circ}\right.$ ), and when the FMS nanoparticles were dispersed into the waterborne polysiloxane latex, the corresponding coating surface turned from
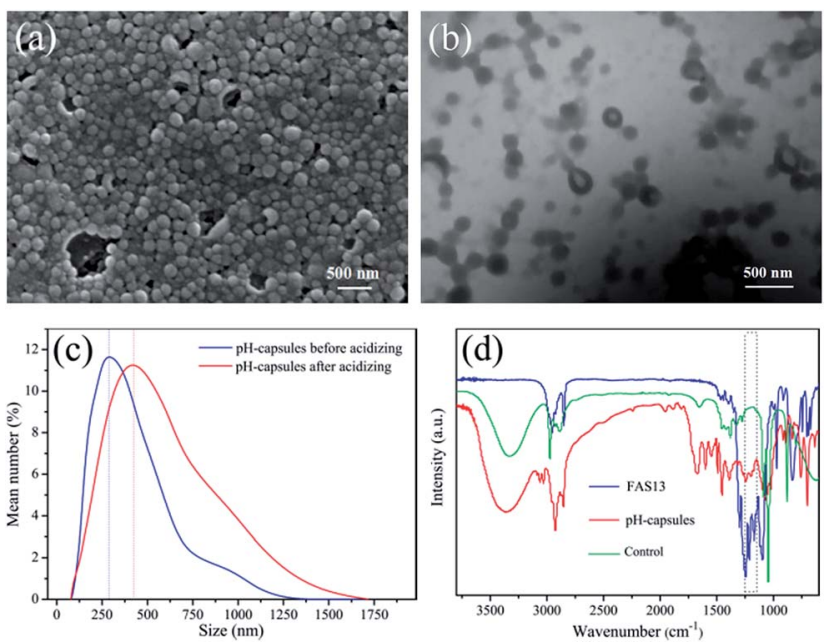

Fig. 1 ( $a$ and b) SEM and TEM micrographs of microcapsules. (c) Particle size distribution of microcapsules measured by DLS. (d) FT-IR spectra of FAS13 and the extracted ethanol of the $\mathrm{pH}$-capsules and control capsules. 
hydrophilicity to hydrophobicity $\left(\mathrm{WCA}=128.1^{\circ}\right)$ at $25 \mathrm{wt} \%$ of FMS (Fig. 2a), which was the critical FMS nanoparticles load and further increasing FMS amount did not cause any obvious increase in WCA. However, when the pH-responsive microcapsules and P25 nanoparticles were added into these coatings (composition: BS45/P25/FMS/pH-capsules = 53/3/25/19 wt/wt), the corresponding formed surface could reach superhydrophobicity $\left(\mathrm{WCA}=155.3^{\circ}\right)$ after $48 \mathrm{~h}$ accelerated weathering test, as shown in Fig. 2b. By comparison, nanocomposite coatings with other particles such as pure P25, P25 plus FMS and P25 plus microcapsules were also checked for their changes of WCA before and after UV irradiation. No obvious changes in WCA were found in pure P25 and P25 plus FMS-based nanocomposite coating, but around $30^{\circ}$ increases were observed for the P25 plus microcapsule-based nanocomposite coatings after accelerated weathering treatment. This indicates that the asobtained superhydrophobic surface is exclusively attributed to the release of FAS13 from microcapsules in the presence of photocatalytic $\mathrm{TiO}_{2}$ nanoparticles. ${ }^{18,38}$ The important point here is that the appropriate content of $\mathrm{pH}$-capsules is very critical. Too many $\mathrm{pH}$-capsules in the coatings would decrease the initial WCA, while too little pH-capsules might affect the durability of superhydrophobic coatings. Moreover, the surface morphologies of the coating before and after UV irradiation were further examined by SEM. As you can see from Fig. 2c, the surface of the coating had a dual-scale roughness with large number of microscale aggregates and nanoscale protuberances,
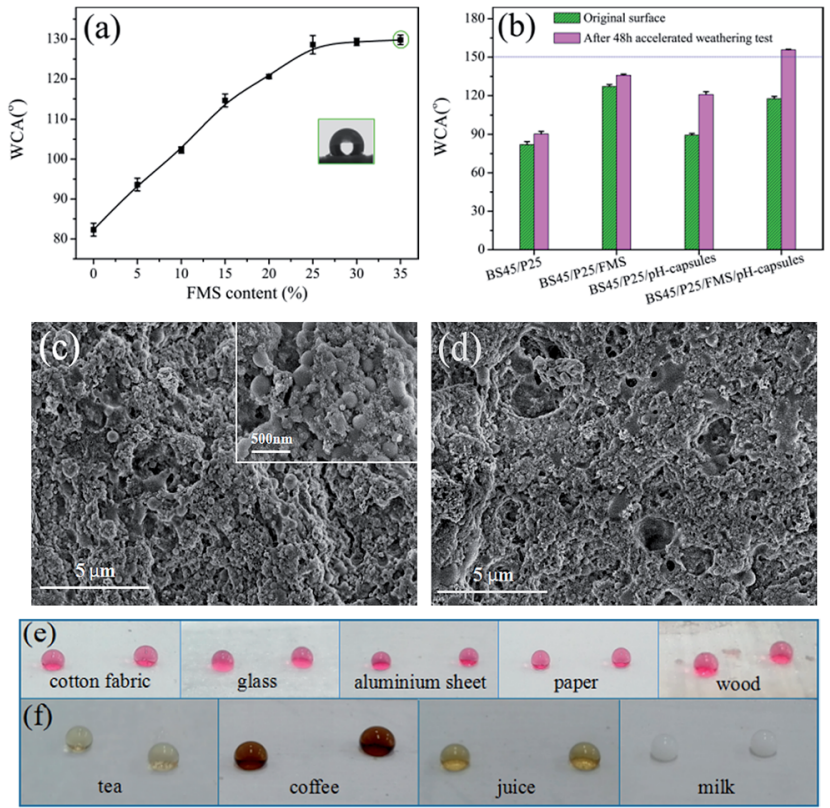

Fig. 2 (a) Variation of WCA with the amount of FMS nanoparticles in the waterborne polysiloxane latex. (b) Changes of WCA of various waterborne coatings before and after UV irradiation in accelerated weathering tester for $48 \mathrm{~h}$. SEM images of the waterborne superhydrophobic coating before (c) and after $48 \mathrm{~h}$ accelerated weathering test (d). (e) Images of the superhydrophobic coatings on different substrates with water droplets on them. (f) Images of the superhydrophobic coatings with various liquids droplets on them. which was mainly composed of the microcapsules and FMS. Nevertheless, the morphology of the coating surface did not changed obviously after UV irradiation (Fig. 2d), illustrating that the superhydrophobicity of the surface was resulted from the releasing of FAS13 in the microcapsules. Moreover, these superhydrophobic surfaces can also be formed on various substrates, e.g., cotton fabric, glass, aluminum plate, paper, and wood, etc. (Fig. 2e). Besides, the coating is also nonwettable by other aqueous liquids such as tea, coffee, orange juice and milk (Fig. 2f).

\subsection{Durability of waterborne superhydrophobic coatings}

As shown in Fig. 3a, water droplets rolled off all the slightly tilted coated samples regardless of composition and surface structure of the substrates. Powdered dirt on the coating could be also efficiently washed off by water, and water droplets completely left the surfaces without wetting or even contaminating the surfaces (Fig. 3b), suggesting that this surface possess a self-cleaning property. This was because the low surface energy substance FAS13 combining with the roughness structure endowed the coating with a weak interaction between
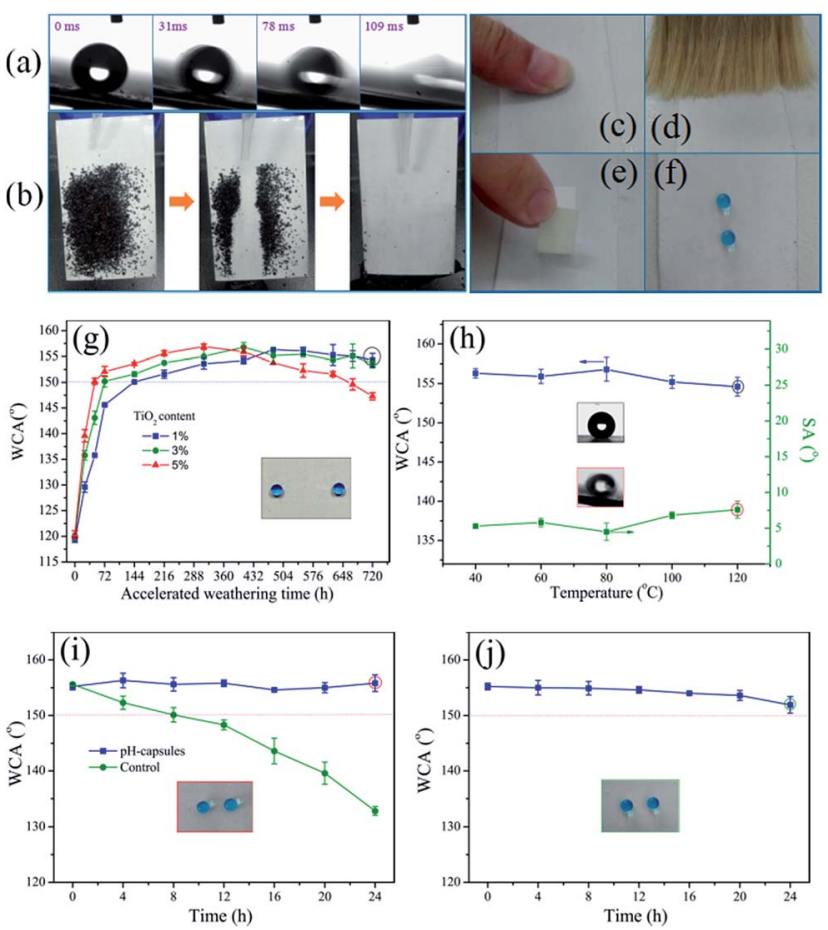

Fig. 3 (a) Photographs of a water droplet $(5 \mu \mathrm{L})$ easily sliding on the $\mathrm{pH}$-capsules based superhydrophobic surface (SA: $6.7^{\circ}$ ). (b) Selfcleaning ability of the $\mathrm{pH}$-capsules based superhydrophobic surface. (c-f) Mechanical stability of the $\mathrm{pH}$-capsules based superhydrophobic surface. (g) The WCA of the $\mathrm{pH}$-capsules superhydrophobic coatings with various $\mathrm{TiO}_{2}$ nanoparticle contents with accelerated weathering time. (h) The WCAs and SAs of coated sample stored in different temperatures from 0 to $120{ }^{\circ} \mathrm{C}$ for $2 \mathrm{~h}$. Variation of WCA of the waterborne superhydrophobic coatings as a function of immersion time in $\mathrm{HCl}$ solution of $\mathrm{pH}=1$ (i), $\mathrm{NaOH}$ solution of $\mathrm{pH}=13$ (j) (the $\mathrm{pH}$ capsules based superhydrophobic coating composition: BS45/P25/ $\mathrm{FMS} / \mathrm{pH}$-capsules $=53 / 3 / 25 / 19 \mathrm{wt} / \mathrm{wt}$ ). 
the surface and the dirt as well as the rolling motion of water droplets on the coating. ${ }^{39}$ Moreover, when the surface of the waterborne superhydrophobic coating was pressed, brushed and taped, this surface could still sustain the superhydrophobicity, manifesting that it has good mechanical stability (Fig. 3c-f). Fig. $3 \mathrm{~g}$ presents the effect of $\mathrm{TiO}_{2}$ nanoparticles content on the superhydrophobic durability of the coatings under UV irradiation. The WCA gradually rose up after aging for all coatings due to photo-degradation of polymer shell. Although the coating with $5 \mathrm{wt} \%$ of $\mathrm{TiO}_{2}$ nanoparticles reached the superhydrophobicity requiring shorter UV irradiation time, the WCA of coatings started to decline after accelerated test for $648 \mathrm{~h}$ and even below $150^{\circ}$ after $720 \mathrm{~h}$. This may be attributed to the photo-induced supra-amphiphilic $\mathrm{TiO}_{2}$ nanoparticles that were uncovered by enough hydrophobic polymer chains. Therefore, the right amount of $\mathrm{TiO}_{2}$ nanoparticles (i.e., 3 $\mathrm{wt} \%$ ) is very important. And besides, thermal stability can also be evaluated by placing the coated sample into invariable temperature environment with different temperature from 0 to $120^{\circ} \mathrm{C}$ for $2 \mathrm{~h}$. The WCA and SA values are shown in Fig. $3 \mathrm{~h}$ with no great change, meaning that the superhydrophobicity of this coating possesses excellent thermal stability. Furthermore, the acid- and alkali-resistant ability of the waterborne superhydrophobic coating was evaluated. Under strong acid situation $(\mathrm{pH}=1)$ for $24 \mathrm{~h}$, the $\mathrm{pH}$-capsules based superhydrophobic coating could maintain its superhydrophobicity compared with non-responsive microcapsules based super-hydrophobic coating. This indicated that the durability of the superhydrophobicity was exclusively attributed to the pH-capsules which can release hydrophobic FAS13 molecules under acidic conditions. Also, this pH-capsules based superhydrophobic coating could withstand the attacks of strong alkali solution $(\mathrm{pH}=13)$ for $24 \mathrm{~h}$ and the WCA values were still greater than $150^{\circ}$. These results illustrated that $\mathrm{pH}$-capsules based superhydrophobic coatings possessed a good resistance to acidic and basic liquids.

\subsection{Self-regeneration of waterborne superhydrophobic coatings upon mechanical damage}

Due to the continuous renewal of the surface, natural superhydrophobic plant leaves or insect wings can maintain their nonwettability even after mechanical damage. ${ }^{7}$ In this study, the sandpaper abrasion test was carried out to evaluate the influence of mechanical abrasion and the self-healing ability of the superhydrophobic coating by using the abrasive sandpaper under $10 \mathrm{kPa}$ pressures (Fig. 4a and b). From Fig. 4c, it can be seen that the WCA of surface obviously declined and the sliding angle rose, indicating that the surface lost its superhydrophobicity owing to the loss of hydrophobic substance on surfaces. Fortunately, the superhydrophobicity of this damaged surface could be gradually regained under UV irradiation. Moreover, using the abrading/UV irradiation healing process could repair the coating for more than seven cycles, as shown Fig. 4 d. In addition, the typical atomic compositions of surfaces before and after self-repairing were measured by XPS (Fig. 4e). After abraded, the F atomic concentration sharply decreased,
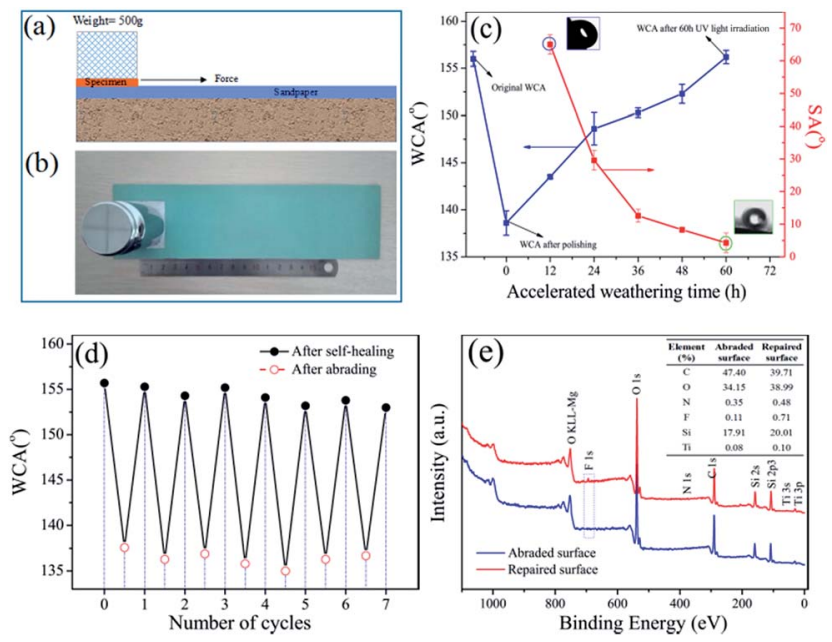

Fig. 4 ( $a$ and b) Schematic illustrations of the sandpaper abrasion test. (c) Changes of WCA and SA of the damaged superhydrophobic surfaces along with accelerated weathering time. (d) Changes of WCA for the superhydrophobic coatings as a function of the repeated cycle number of the abrading under $10 \mathrm{kPa}$ pressures and accelerated weathering. (e) XPS analysis of the surface of superhydrophobic coatings before and after self-healing (the $\mathrm{pH}$-capsules based superhydrophobic coating composition: BS45/P25/FMS/pH-capsules = 53/ 3/25/19 wt/wt).

suggesting the loss of hydrophobic substance on surfaces. However, the $\mathrm{F}$ atomic concentration increased after UVirradiation (from $0.11 \%$ to $0.71 \%$ ), directly indicating that FAS13 molecules have been released from $\mathrm{pH}$-capsules and enriched at the top surfaces of the coatings. Meanwhile, the $\mathrm{O} / \mathrm{C}$ atomic ratio considerably increased, which resulted from the degradation of polymer of $\mathrm{TiO}_{2}$ nanoparticles under UV irradiation. These results demonstrated that the as-obtained superhydrophobic coating had excellent self-healing ability upon mechanical damage.

\subsection{Recovery of waterborne superhydrophobic coatings after oil pollution}

However, superhydrophobic surfaces would be contaminated by oily organic contaminants in outdoors, and thus lose their self-cleaning ability. ${ }^{7,18}$ Herein, we examined the resistance of the superhydrophobic coating to organic contaminants, using salad oil as model pollutants. As shown in Fig. 5a, the WCA of superhydrophobic coating decreases from 155.6 to $61.2^{\circ}$ after casting a thin layer of salad oil. However, the coating surface recovers its superhydrophobicity $\left(151.2^{\circ}\right)$ after accelerated weathering test for $48 \mathrm{~h}$. In contrast to control experiments, when the pH-capsules and the FMS without $\mathrm{TiO}_{2}$ were mixed with polysiloxane latex, its coating surface changed from hydrophobic to hydrophilic state after cast by salad oil, and kept this hydrophilicity even after accelerated weathering test for $72 \mathrm{~h}$, suggesting that the surface $\mathrm{TiO}_{2}$ nanoparticles of $\mathrm{pH}$ capsules in the coating can photocatalytically also decompose the salad oil except decomposing polymer shell under UV light and therefore endow the coatings with the resistance to organic contaminants. Even after five cycles of salad oil casting and UV 

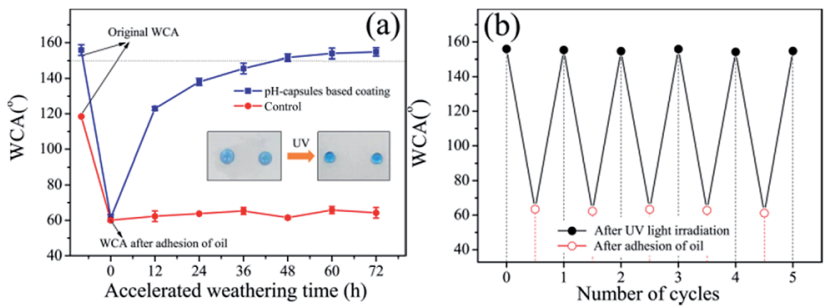

Fig. 5 (a) Changes of WCA for the pH-capsules based superhydrophobic coating and control coating as a function of UV irradiation time, the surfaces were cast by a thin layer of salad oil. (b) Changes of WCA for $\mathrm{pH}$-capsules based superhydrophobic coatings as a function of the number of the repeated cycles of salad oil adhesion and accelerated weathering (the $\mathrm{pH}$-capsules based superhydrophobic coating composition: BS45/P25/FMS/pH-capsules = 53/3/25/19 wt/ wt).

illumination in the accelerated weathering tester, this coating still remained its superhydrophobic state (Fig. 5b). All these results indicated that the as-obtained superhydrophobic coatings can decompose organic contaminants and exhibit excellent self-recovering and self-cleaning ability.

\subsection{Self-repairability of waterborne superhydrophobic coatings under corrosive environment}

Because superhydrophobic coatings can put off water diffusion, it can also protect the metal substrates from corroding effectively. However, when the superhydrophobic coating applied in a high-salt environment, the saltwater would inevitably permeate the coating and result in the decrease of its superhydrophobicity, and it might lose protectiveness against corrosion. ${ }^{40-42}$ Therefore, the chemical stability of coating was evaluated by measuring the WCAs after immersing into saltwater at room temperature. As shown in Fig. 6a-c, the WCAs of coatings decreased slightly after immersion in a $5 \mathrm{wt} \% \mathrm{NaCl}$ aqueous solution for $24 \mathrm{~h}$ and the surface still kept highhydrophobicity $\left(136^{\circ}\right)$. And fortunately, the surface could restore to its original superhydrophobicity after UV irradiation while the control coating had not recovered the superhydrophobicity due to no P25 in the coatings. Moreover, these coatings still keep their superhydrophobicity even after the cycles of $\mathrm{NaCl} / \mathrm{UV}$ or $\mathrm{NaCl} /$ acid healing process for 5 times (Fig. 6d and e). These results indicate that the restoration of the superhydrophobic property results from FAS13 releasing of the microcapsules under UV or pH stimuli.

\subsection{Anti-icing performance of waterborne superhydrophobic coatings}

However, superhydrophobic coatings in the natural environment always need to face some extreme weather such as frost and freezing, which will negatively affect the coating's lifetime. We further have done the anti-icing tests for this superhydrophobic coating. As shown in Fig. $7 \mathrm{a}$ and $\mathrm{b}$, the water droplet was spread out on a bare aluminum sheet with the ice forming completely for about $19 \mathrm{~min}$ and could hardly peel from the bare aluminum sheet. However, the iced water droplet
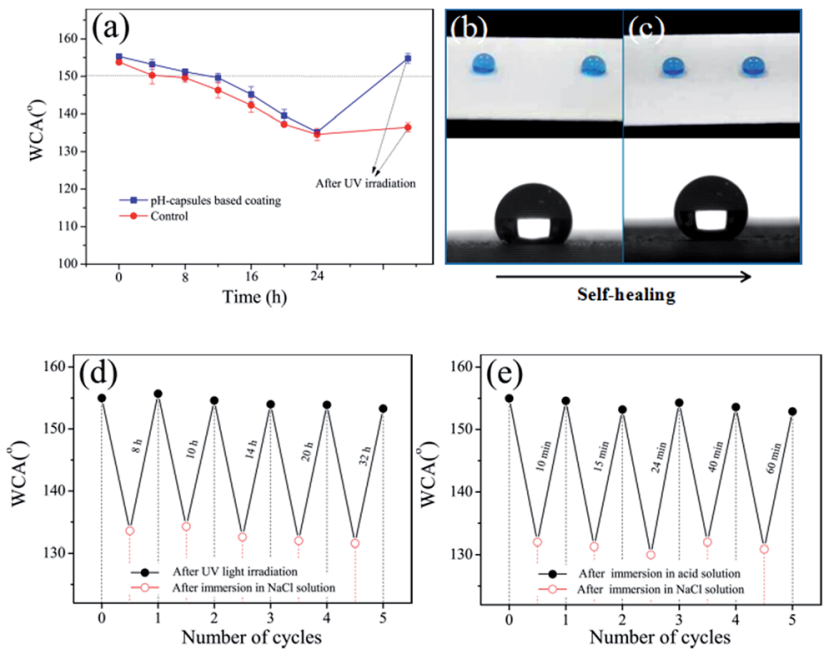

Fig. 6 (a) Changes of WCA for the $\mathrm{pH}$-capsules based superhydrophobic coating and control coating after immersed in 5 wt\% $\mathrm{NaCl}$ aqueous solution for $24 \mathrm{~h}$ and after UV irradiation. Photographs of water droplets and contact angles on the superhydrophobic surfaces, (b) after immersed in $5 \mathrm{wt} \% \mathrm{NaCl}$ aqueous solution for $24 \mathrm{~h}$, (c) after accelerated weathering test for $32 \mathrm{~h}$. (d) Change of WCAs for the superhydrophobic coatings as a function of repeated immersing and accelerated weathering cycles. (e) Change of WCAs for the superhydrophobic coatings as a function of repeated immersing and acid treating cycles (the $\mathrm{pH}$-capsules based superhydrophobic coating composition: BS45/P25/FMS/pH-capsules = 53/3/25/19 wt/wt).

could easily separate itself from the coated aluminum sheet, and the icing process of the water droplet on the coated aluminum sheet was about $40 \mathrm{~min}$, which is 2 times longer than the control sample (Fig. $7 \mathrm{c}$ and d). In theory, the water droplet on superhydrophobic surface has a smaller contact area with the substrate, which provided less possible area of heterogeneous nucleation. On the other hand, air pockets also can hinder the heat transfer between the water droplet and the substrate, which can prolong the icing time. ${ }^{39}$ This phenomenon also demonstrated low adhesive force of coating to ice. As a whole, this superhydrophobic coating can not only delay icing time but also reduce the adhesive force between ice and substrate. Furthermore, although the adhesive force between the abraded superhydrophobic coating and ice increased due to the loss of its superhydrophobicity (Fig. 7e and f), the ice droplet could easily peel from the damaged surface after $36 \mathrm{~h} \mathrm{UV}$ irradiation (Fig. $7 \mathrm{~g}$ and $\mathrm{h}$ ), suggesting the anti-icing property of this coating has been repaired as its superhydrophobicity restored. Moreover, the ice adhesion strength between iced water and the samples were also tested. As shown in Fig. 7i, the results showed that the superhydrophobic coating had the lowest adhesion strength, 0.09 MPa, this value of the adhesion strength was lower than the adhesion strength of aluminium plate. When the superhydrophobic coating was abraded, its adhesion strength increased. However, the adhesion strength was down after the abraded coating self-repairing. These results can also be consistent with the phenomena of the iced water droplet separating itself from the coated aluminum sheet. In order to fully study the anti-icing process, the periodic icing/melting 

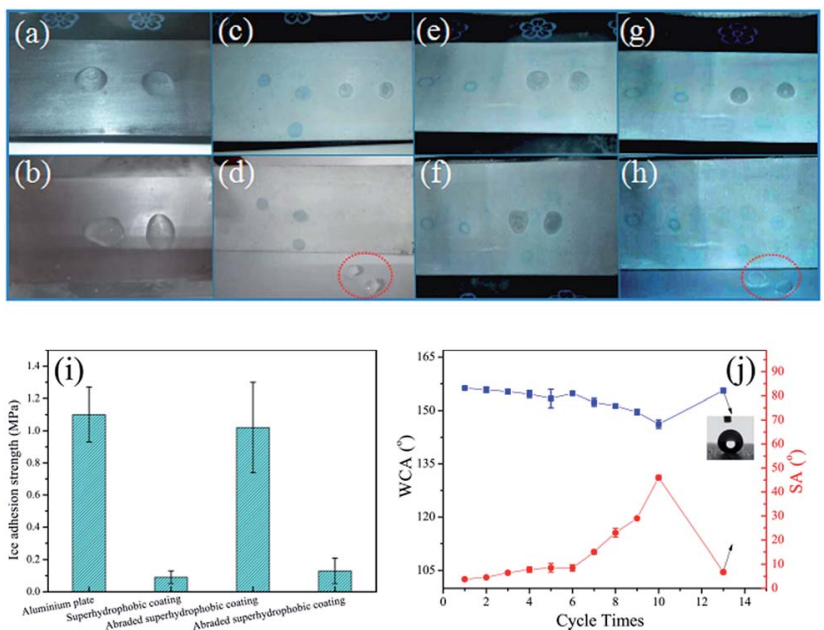

Fig. 7 Photographs of anti-icing test of pure aluminium plate (a), the $\mathrm{pH}$-capsules based superhydrophobic coating (c), abraded $\mathrm{pH}$ capsules based superhydrophobic coating (e) and repaired $\mathrm{pH}$ capsules based superhydrophobic coating (g). The attachment and separation of iced water droplet on aluminium plate (b), the $\mathrm{pH}$ capsules based superhydrophobic coating (d), abraded pH-capsules based superhydrophobic coating (f) and repaired $\mathrm{pH}$-capsules based superhydrophobic coating (h). (i) The ice adhesion strength of the samples: aluminium plate; the $\mathrm{pH}$-capsules based superhydrophobic coating; abraded $\mathrm{pH}$-capsules based superhydrophobic coating and repaired $\mathrm{pH}$-capsules based superhydrophobic coating. (j) The WCA and $\mathrm{SA}$ as a function of icing/melting cycle times for the $\mathrm{pH}$-capsules based superhydrophobic coating (the $\mathrm{pH}$-capsules based superhydrophobic coating composition: BS45/P25/FMS/pH-capsules = 53/ 3/25/19 wt/wt).

process was carried out. As shown in Fig. 7j, the WCA of superhydrophobic surface slightly decreased while the SA rose obviously after 10 times icing/melting, which might be resulted from the reduction of low surface energy materials in the surface. When this coating was further irradiated by UV light in the accelerated weathering tester, the surface gradually recovered its superhydrophobicity (WCA: $153^{\circ}$, SA: $6.7^{\circ}$ ) and again took on the anti-icing property. All these results illustrated that the as-obtained superhydrophobic coatings exhibited good antiicing durability.

\section{Conclusions}

In summary, we have successfully prepared UV and $\mathrm{pH}$ stimuliresponsive self-repairing waterborne superhydrophobic coatings from $\mathrm{pH}$-responsive microcapsules and photocatalytic $\mathrm{TiO}_{2}$ nanoparticles. The as-obtained coatings can be readily sprayed on various substrates to form superhydrophobic surfaces after UV-irradiation due to the UV-inducing release of FAS13, and their surfaces showed remarkable robustness against UV light and acidic and basic liquids. In particular, the surfaces can recover their superhydrophobic and self-cleaning ability under UV light even after mechanically damaged or polluted with organic contaminants. Furthermore, this superhydrophobic coating also has the self-repairability even after being immersed into $\mathrm{NaCl}$ aqueous solutions for $24 \mathrm{~h}$ or ten icing/melting cycles. It is believed that the UV light and pH dual-inducing self-repairing and self-cleaning ability as well as the waterborne character of the coatings assured their great practicality outdoors.

\section{Acknowledgements}

Financial supports were received from the Natural Science Foundation of Jiangsu Province (BK20160186), the China Postdoctoral Science Foundation (No. 2016T90418 and 2016M600358), the Fundamental Research Funds for the Central Universities (No. JUSRP116017) and the Priority Academic Program Development of Jiangsu Higher Education Institutions.

\section{References}

1 A. Lafuma and D. Quéré, Nat. Mater., 2003, 2, 457-460.

2 S. Wang and L. Jiang, Adv. Mater., 2007, 19, 3423-3424.

3 K. Liu, X. Yao and L. Jiang, Chem. Soc. Rev., 2010, 39, 32403255.

4 B. Bhushan and Y. C. Jung, Prog. Mater. Sci., 2011, 56, 1-108.

$5 \mathrm{H}$. Bellanger, T. Darmanin, E. Taffin de Givenchy and F. Guittard, Chem. Rev., 2014, 114, 2694-2716.

6 J. Zimmermann, F. A. Reifler, G. Fortunato, L. Gerhardt and S. Seeger, Adv. Funct. Mater., 2008, 18, 3662-3669.

7 T. Verho, C. Bower, P. Andrew, S. Franssila, O. Ikkala and R. H. A. Ras, Adv. Mater., 2011, 23, 673-678.

8 H. Wang, Y. Xue, J. Ding, L. Feng, X. Wang and T. Lin, Angew. Chem., Int. Ed., 2011, 50, 11433-11436.

9 X. Deng, L. Mammen, H. Butt and D. Vollmer, Science, 2012, 335, 67-70.

10 X. Zhou, Z. Zhang, X. Xu, F. Guo, X. Zhu, X. Men and B. Ge, ACS Appl. Mater. Interfaces, 2013, 5, 7208-7214.

11 K. Chen, Y. Wu, S. Zhou and L. Wu, Macromol. Rapid Commun., 2016, 37, 463-485.

12 Y. Li, L. Li and J. Sun, Angew. Chem., 2010, 122, 6265-6269.

13 X. Wang, X. Liu, F. Zhou and W. Liu, Chem. Commun., 2011, 47, 2324-2326.

14 T. K. Wong, S. H. Kang, S. K. Y. Tang, E. J. Smythe, B. D. Hatton, A. Grinthal and J. Aizenberg, Nature, 2011, 477, 443-447.

15 L. Ionov and A. Synytska, Phys. Chem. Chem. Phys., 2012, 14, 10497-10502.

16 C. Xue and J. Ma, J. Mater. Chem. A, 2013, 1, 4146-4161.

17 H. Zhou, H. Wang, H. Niu, A. Gestos and T. Lin, Adv. Funct. Mater., 2013, 23, 1664-1670.

18 K. Chen, S. Zhou and L. Wu, Chem. Commun., 2014, 50, 11891-11895.

19 K. Chen, S. Zhou and L. Wu, ACS Nano, 2016, 10, 1386-1394. 20 Y. Li, S. Chen, M. Wu and J. Sun, Adv. Mater., 2014, 26, 33443348.

21 A. C. C. Esteves, Y. Luo, M. W. P. van de Put, C. C. M. Carcouët and G. de With, Adv. Funct. Mater., 2014, 24, 986-992.

22 U. Manna and D. M. Lynn, Adv. Mater., 2013, 25, 5104-5108.

23 H. Zhou, H. Wang, H. Niu, A. Gestos, X. Wang and T. Lin, Adv. Mater., 2012, 24, 2409-2414. 
24 B. Li, S. Chen and J. Zhang, J. Appl. Polym. Sci., 2014, 131, 39708.

25 Y. Shi, X. Gao, D. Zhang, Y. Liu and G. Huang, RSC Adv., 2014, 4, 41453-41460.

26 Y. Liu, Y. Shi, D. Zhang, J. Li and G. Huang, Polymer, 2013, 53, 6140-6149.

27 T. Lv, Z. Cheng, E. Zhang, H. Kang, Y. Liu and L. Jiang, Small, 2016, DOI: $10.1002 / \mathrm{smll} .201503402$.

28 Q. Rao, K. L. Chen and C. Wang, RSC Adv., 2016, 6, 5394953954.

29 J. E. Mates, T. M. Schutzius, I. S. Bayer, J. Qin, D. E. Waldroup and C. M. Megaridis, Ind. Eng. Chem. Res., 2014, 53, 222-227.

30 J. E. Mates, R. Ibrahim, A. Vera, S. Guggenheim, J. Qin, D. Calewarts, D. E. Waldroupd and C. M. Megaridis, Green Chem., 2016, 18, 2185-2192.

31 T. Chen, P. J. Colver and S. A. F. Bon, Adv. Mater., 2007, 19, 2286-2289.

32 M. F. Haase, D. O. Grigoriev, H. Möhwald and D. G. Shchukin, Adv. Mater., 2012, 24, 2429-2435.

33 R. Kurapatia and A. M. Raichur, Chem. Commun., 2013, 49, 734-736.
34 G. Wu, J. An, X. Tang, Y. Xiang and J. Yang, Adv. Funct. Mater., 2014, 24, 6751-6761.

35 Y. Cong, K. Chen, S. Zhou and L. Wu, J. Mater. Chem. A, 2015, 3, 19093-19099.

36 Y. Long, C. Liu, B. Zhao, K. Song, G. Yang and C. Tung, NPG Asia Mater., 2015, 7, e148.

37 K. Chen, S. Zhou, S. Yang and L. Wu, Adv. Funct. Mater., 2015, 25, 1035-1041.

38 I. Sopyan, M. Watanabe, S. Murasawa, K. Hashimoto and A. J. Fujishima, Electroanal. Chem., 1996, 415, 183-186.

39 Y. Si, Z. Guo and W. Liu, ACS Appl. Mater. Interfaces, 2016, 8, 16511-16520.

40 D. Zhang, H. Qian, L. Wang and X. Li, Corros. Sci., 2016, 103, 230-241.

41 P. Phanthong, G. Guan, S. Karnjanakom, X. Hao, Z. Wang, K. Kusakabe and A. Abudula, RSC Adv., 2016, 6, 1332813334.

42 L. B. Boinovich, A. M. Emelyanenko, K. A. Emelyanenko and K. I. Maslakov, Phys. Chem. Chem. Phys., 2016, 18, 3131-3136. 